\title{
\#cutting: Non-suicidal self-injury (NSSI) on Instagram
}

\author{
R. C. Brown ${ }^{1 *}$, T. Fischer ${ }^{2}$, A. D. Goldwich ${ }^{3}$, F. Keller ${ }^{1}$, R. Young ${ }^{4}$ and P. L. Plener ${ }^{1}$ \\ ${ }^{1}$ Department for Child and Adolescent Psychiatry and Psychotherapy, University Hospital Ulm, Ulm, Germany \\ ${ }^{2}$ Freelancing data journalist, Berlin, Germany \\ ${ }^{3}$ Freelancing software developer, Berlin, Germany \\ ${ }^{4}$ MRC Social and Public Health Sciences Unit, University of Glasgow, Glasgow, Scotland
}

Background. Social media presents an important means for social interaction, especially among adolescents, with Instagram being the most popular platform in this age-group. Pictures and communication about non-suicidal self-injury (NSSI) can frequently be found on the internet.

\begin{abstract}
Methods. During 4 weeks in April 2016, $n=2826$ (from $n=1154$ accounts) pictures which directly depicted wounds on Instagram were investigated. Those pictures, associated comments, and user accounts were independently rated for content. Associations between characteristics of pictures and comments as well as weekly and daily trends of posting behavior were analyzed.

Results. Most commonly, pictures depicted wounds caused by cutting on arms or legs and were rated as mild or moderate injuries. Pictures with increasing wound grades and those depicting multiple methods of NSSI generated elevated amounts of comments. While most comments were neutral or empathic with some offering help, few comments were hostile. Pictures were mainly posted in the evening hours, with a small peak in the early morning. While there was a slight peak of pictures being posted on Sundays, postings were rather evenly spread across the week.

Conclusions. Pictures of NSSI are frequently posted on Instagram. Social reinforcement might play a role in the posting of more severe NSSI pictures. Social media platforms need to take appropriate measures for preventing online social contagion.
\end{abstract}

Received 15 December 2016; Revised 29 May 2017; Accepted 2 June 2017; First published online 14 July 2017

Key words: Instagram, non-suicidal self-injury, NSSI, social contagion, social media.

\section{Introduction}

The social network 'Instagram' has been rated as the most important network among adolescents in the USA (Guimaraes, 2014) with most Instagram users being around high-school age (Duggan \& Smith, 2013). In 2015, Instagram was the second most popular social media platform among German adolescents (after YouTube) with $44 \%$ reporting Instagram to be an important part of their daily lives (Feierabend et al. 2015). In January 2015, around 11 million photographs were tagged with \#depression (\#happiness: 35 million) on Instagram (Fischer et al. 2015). Based on Instagram data from May 2014, approximately 27\% of \#depression pictures are associated with the hashtag \#cutting, the second biggest specific \#depression co-occurring hashtag after \#ana ('anorexia', 31\%)

\footnotetext{
* Address for correspondence: R. C. Brown, M.Sc., Department for Child and Adolescent Psychiatry and Psychotherapy, University

Hospital Ulm, Steinhoevelstr. 5, 89075 Ulm, Germany.

(Email: rebecca.brown@uniklinik-ulm.de)
}

(Fischer et al. 2015). A similar pattern was found in another study analyzing depression-related hashtags on Instagram (Andalibi et al. 2017).

In systematic reviews, several potential risks and benefits of online activity related to non-suicidal selfinjury (NSSI) were identified (Dyson et al. 2016; Lewis \& Seko, 2016). Potential benefits included a reduction of social isolation by providing a sense of community, encouragement for recovery, reducing NSSI urges, and emotional self-disclosure, while potential risks included triggering NSSI urges (including live demonstrations of NSSI in videos), normalization, acceptance or stigmatization of NSSI and NSSI (social) reinforcement. This was also shown in a study by Baker \& Lewis (2013) showing that while some participants reported a reduction of loneliness after viewing NSSI pictures, others reported reinforcing and encouraging effects. NSSI social reinforcement has been a subject of research in a large number of studies. However, these effects have mostly been evaluated in direct social contact, rather than online social interaction. Besides positive and negative 
automatic reinforcement (i.e. reduction of negative feeling states or evoking positive feelings), Nock \& Prinstein's (2004) well-established model of the functionality of NSSI states social reinforcement as an important factor in NSSI. Posting NSSI pictures online might also serve a positive social reinforcement function through receiving direct feedback for NSSI pictures in the form of comments or likes, although posting NSSI content is not the same as NSSI acts in itself.

The content of online NSSI material has only been analyzed in few studies. In a study reviewing YouTube videos, Lewis et al. (2011) found that viewers rated videos with NSSI content very positively. Around half of the videos were educational or factual, while the other half contained a melancholic tone. Almost all videos showed explicit NSSI photographs, and one third of videos including video footage of persons, also showed a live depiction of NSSI. In a qualitative analysis of 516 pictures posted on the platform Flickr, Seko (2013) found most pictures to be candid, not aestheticized colored depictions of wounds or scars. Most wounds looked superficial, without causing much damage to body tissue. While two thirds of pictures captured scars that were completely healed up, around one third showed fresh wounds. However, a large percentage of pictures showed wounds in several stages of healing (i.e. fresh wounds on top of old scars). In a study using the hashtag \#selfharmmm, a popular hashtag on Instagram for NSSI, Moreno et al. (2015) identified different hashtags commonly used in association with NSSI pictures (i.e. \#cat, \#selfinjuryyy, \#blithe) and mechanisms at work in the network. Only in around one third of NSSI related hashtags, content advisory warnings were generated.

The current study includes a comprehensive analysis of NSSI pictures published on Instagram over the course of 4 weeks in spring 2016, which were associated with the most common German self-injury hashtags. Due to the lack of former studies, and due to the fact that this study is the first to investigate NSSI content in a German speaking network, our approach is mainly explorative and descriptive. The first aim of this study was to systematically describe the extent of NSSI on Instagram in a German speaking population, as no such results have yet been published and Instagram represents one of the major online social networks in Germany. According to results of studies on other social media networks we hypothesized that there would be specific NSSI pictures, which would generate positive and negative comments alike. The second aim was to describe online content of German speaking users, as most research so far has been limited to English speaking populations. As users were thus limited to one time-zone, this study is the first to include timely calculations of postings, which is not possible when including only English hashtags. As no such research has been possible in other studies, this was a purely explorative approach. This approach also allowed us to investigate the possible effect of social reinforcement further, which is an important topic in NSSI research, and systematic research on the reinforcing effect of online content is very sparse. As results from previous research point towards a possible socially reinforcing effect of NSSI content online, we expected to find positive associations of pictures and comments being posted.

\section{Methods}

German hashtags were chosen, as Germany shows one of the highest rates of NSSI among adolescents. Furthermore a major advantage using German hashtags is the references are more location specific than using English hashtags, which would have included a wide range of international users. In a first step, all pictures with the hashtag \#ritzen ('cutting') - which is the most popular hashtag for NSSI in Germany (Fischer et al. 2015) - were downloaded for $48 \mathrm{~h}$ via Instagram's API from users with a profile accessible to the public. This yielded 1135 pictures. The 30 most common German hashtags associated with those pictures were identified, as they were used at least twice. Pictures associated with those 30 hashtags were then downloaded for $72 \mathrm{~h}$, which yielded 5588 pictures. Two independent raters defined whether those pictures focused directly on a wound or scar $(\kappa$ $=0.88$ ). A total of 293 pictures meeting these criteria were identified. Hashtags within which at least $5 \%$ of the pictures focused on depicting wounds or scars were included in the final download. These hashtags were \#klinge ('blade'), \#narben ('scars'), \#selbstverletzung ('self-injury'), \#ritzen ('cutting'), \#suizidgedanken ('suicidal thoughts'), \#klingenliebe ('blade-love'), \#blut ('blood'), \#svv (acronym for self-injurious behavior), \#selbsthass ('self-hate'), \#depressiv ('depressive'), \#depressionen ('depressions'), \#suizid ('suicide'), \#ritzengegendenschmerz ('cutting against the pain'), \#selbstmord ('suicide'), \#depri ('colloquial for depressed'), and \#sterben ('dying').

All pictures and user accounts associated with those hashtags were downloaded at an hourly rate during 4 weeks in April 2016. Gender and age of 1154 users who had posted NSSI pictures were taken from their profiles (if applicable). However, characteristics of users were excluded from further analyses, since most profiles were anonymous and users' indication of age and gender could not be verified (for details see below). The numbers of 'likes' and comments for every picture were counted and the time and date of 
when each picture was posted was recorded. However, the number of 'likes' was not included in the final analyses, due to rather high correlation with the number of followers a user had (Spearman- $\rho=0.46, p<0.001$ ) and may therefore have been confounded. The number of comments was less strongly correlated with the number of followers (Spearman- $\rho=0.24, p<0.001$ ).

In order to compare timely trends with more neutral posts than NSSI pictures, all pictures which were geotagged in Berlin during 4 weeks in April 2015 were used as a reference for hourly Instagram activity.

The study was approved by the IRB of the University of Ulm. Data were collected through the public Instagram API (https://www.instagram.com/ developer) and are securely stored in an internal database. Access to the data is restricted to avoid personal identification of users and to comply with Instagram Terms of Use (https://www.instagram.com/about/ legal/terms/) and API Terms (https://www.instagram. com/about/legal/terms/api/).

\section{Rating of pictures and comments}

For the coding of pictures detailed instructions, which included example images for each category, were defined. Studies investigating NSSI content online were screened (i.e. Lewis et al. 2011; Seko, 2013) and the most feasible categories for the current research questions and large amount of data were chosen. After an initial round of coding by two undergraduate psychologists, which yielded rather low inter-rater-reliabilities, those initial codings were screened for potential sources of error by the authors, yielding problems with coding instructions. Finally, the instructions were refined and coding was repeated. Final coding was performed independently by two authors of this paper with several years of experience in the field of NSSI. All pictures were rated by those two independent raters for content (wound/scar v. no wound/scar), wound grade (mild, moderate, severe), type of wound (e.g. cut, scar, bruise, burn), body region (upper extremities, lower extremities, torso, head, neck) and object (razor blade, shard, coin, etc.), if depicted. According to Landis \& Koch (1977), inter-rater-reliability was almost perfect for content $(\kappa=$ $0.95)$, body region $(\kappa=0.83)$, object $(\kappa=0.87)$, wound grade $(\kappa=0.88)$, and type of wound $(\kappa=0.93)$. Pictures directly depicting wounds are labeled 'NSSI pictures' in the following.

A total of 8154 comments was identified and analyzed for NSSI pictures downloaded in the first 2 weeks of April. Only comments by other users (excluding self-referential comments) were rated $(n=6651)$. Content of comments was assigned to one of the following categories: complimenting on the wound (e.g. 'This looks nice'), empathetic reaction (e.g. 'I know how you feel'), offering help (e.g. 'if you need me, I'm always there for you'), warning/asking user to stop behavior (e.g. 'please stop'), abuse ('why don't you go and kill yourself'), and discussion (comment not directed at the user/picture). Inter-rater-reliability for comments was moderate $(\kappa=0.44)$.

Conflicting ratings were decided by a third rater taking the original ratings into account. Calculations were conducted based on these ratings. It became clear in the process that inter-rater reliability was mostly decreased by the fact that scars resulting from cuts and fresh cuts were often not clearly distinguishable. Therefore, wounds and scars resulting from cutting were identified as 'cutting' for further analyses.

Pictures, which were obviously copied (e.g. popular pictures that were posted from different accounts, or pictures taken from movies) or where the wound was not focus of the picture (e.g. text or poem written over the picture, making the wound the background), as well as drawings of wounds were not included in the analyses.

\section{Statistical analyses}

Statistical analyses were performed using the statistics software IBM SPSS Statistics 21 and using $R$, a software for statistical computing and graphics (R Development Core Team, 2008). Inter-rater-reliability (Cohen's $\kappa$ ) was calculated. For group comparisons, analyses of variance (ANOVAs) and $t$ tests were calculated, where applicable. Spearman rank correlation coefficients were calculated for associations between timespans between pictures and number of comments a picture had received. Concerning the frequency distribution of wound pictures within the stream of pictures, the distribution of random occurrences was determined empirically. The $R$ function runif was used to generate a sequence of 300000 uniformly distributed random numbers between 0 and 1 , and, given a base rate of $8.8 \%$ wound pictures, each number $<0.088$ was coded as a 'wound picture'. The resulting distribution was approximated by a (well fitting) exponential function (cf. Fig. 3).

\section{Results}

A total number of 32182 pictures from 6721 user accounts were posted during 4 weeks in April 2016, using the most common German hashtags for NSSI. $2826(8.8 \%)$ of these pictures contained NSSI content coming from 1154 accounts. NSSI ratio (number of pictures directly showing NSSI compared with the total amount of pictures) varied between $5.7 \%$ (\#sterben) and $16.4 \%$ (\#selbstverletzung) between hashtags. 


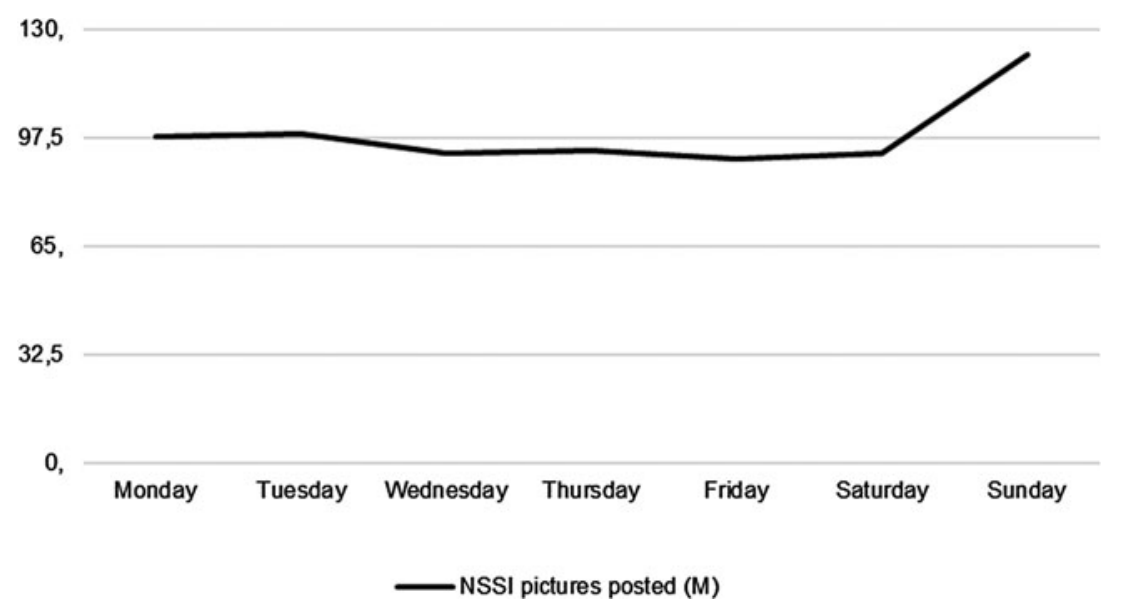

Fig. 1. Average amount of pictures being posted on each day of the week (across all 4 weeks).

\section{Characteristics of users}

A large majority of user profiles in which NSSI pictures (at least one) had been posted were anonymous ( $n=$ $958,83.0 \%$ ), meaning they did not reveal their name, depict their face, or provide other identifying information. In $18.8 \%(n=217)$ of all NSSI-related profiles, faces were clearly shown on their account. A small minority $(n=9,0.7 \%)$ additionally identified their environment (e.g. friends, family, place of residency). Of those users whose gender was identifiable (71\%, $n=819$ ), the vast majority indicated to be female (91\%, $n=745)$ compared with those who identified themselves as male $(9 \%, n=74)$. A total of $n=480$ users $(41.6 \%)$ indicated an age on their profile page. Users' stated age ranged from 12 to 21 (the minimum age for using Instagram is officially 13 years), with an average of 14.8 years (S.D. $=1.6$ ).

\section{Characteristics of pictures}

Of the $n=2826$ pictures, which directly depicted wounds, $39.6 \%$ of wounds were rated as mild (i.e. superficial scratches), $47.8 \%$ were rated as moderate (i.e. deeper cut, blood flowing), $12.6 \%$ were rated as severe (i.e. very deep, gaping cut or a very large amount of deeper cuts and blood).

The vast majority (93.1\%) of NSSI pictures depicted cuts. Other types of self-injury were less common. Bruises were shown in $1.2 \%$ of pictures, while biting $(0.3 \%)$, burning $(0.4 \%)$, and skin picking $(0.4 \%)$ were depicted less often. Other pictures showed undefinable wounds $(0.3 \%)$ or various combinations of several types of injuries $(1.8 \%$, i.e. cutting and bruises, or cutting and burning in one picture).

Regarding body regions, most pictures showed wounds on upper extremities $(59.6 \%), 8.5 \%$ showed wounds on lower extremities, $2.2 \%$ a combination of lower and upper extremities and 1.7\% depicted wounds located on the torso. Wounds on the head $(0.3 \%)$ and the neck $(0.2 \%)$ were very seldom depicted. In $27.2 \%$ of the pictures, the body region could not be defined.

Objects for NSSI along with the corresponding wound were only shown in $5.8 \%$ of the pictures. Most commonly, razor blades $(4.0 \%)$ or other blades $(1.3 \%)$ were depicted, followed by fingernails, knives and ropes (0.1-0.2\%, respectively).

In 34 instances text was cut into the skin, with one of these words unreadable. These were 'HATE ME / MYSELF (6 times), 'FAT' (5 times), 'FUCK YOU' (3 times), 'alone', 'FAIL', 'FAKE', 'ICH LIEBE DICH' (German for: I love you), 'M', 'SUIZID' (German for: suicide), 'WERTLOS' (German for: worthless), 'DREAM', 'Hot', 'MAX', 'I'm FINE', 'LONELY', 'PAIN', 'NICO', 'STOP EATING', 'ENGEL' (German for: angel), 'NEED HIM', 'WHY', 'MXLi' (each of these words seen in one instance).

\section{Daily trends of numbers of pictures being uploaded}

Pictures depicting NSSI were spread relatively even over the week $(F=1.4, p>0.05)$, though showing peaks on Sundays $(M=122.5)$ and lowest rates on Fridays $(M=91.0$; see Fig. 1$)$.

\section{Hourly trends of pictures of NSSI being uploaded}

The largest peak of pictures with NSSI content being posted was in the evening between 20:00 and 22:00 hours. This was true for weekdays and weekends. After a peak in the early morning on weekdays, there was a clear decline of pictures being posted in the later morning hours during weekdays, but not on the weekend (for details see Fig. 2). When comparing hourly trends of NSSI pictures to all pictures being 


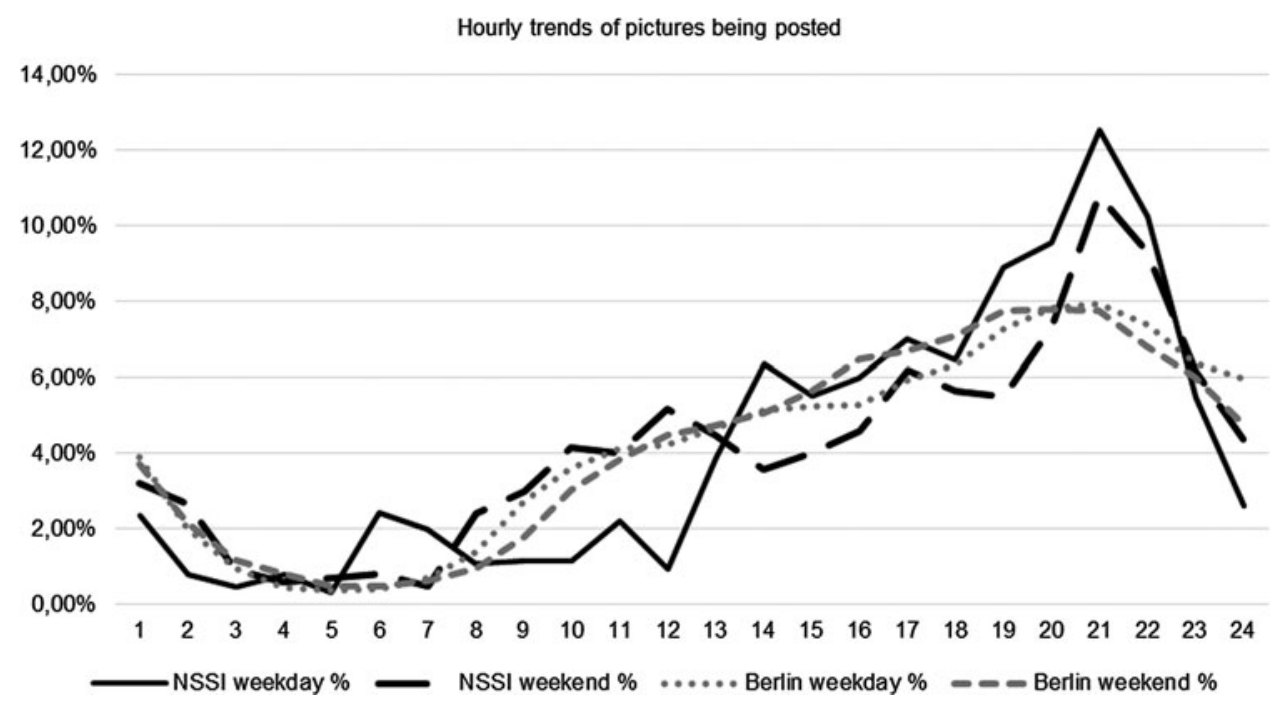

Fig. 2. Hourly trends of percentages of pictures with NSSI content being posted (average of total numbers for weekdays from Monday to Friday and weekends consisting of Saturday and Sunday) and pictures being geotagged in Berlin during 4 weeks in April 2015.

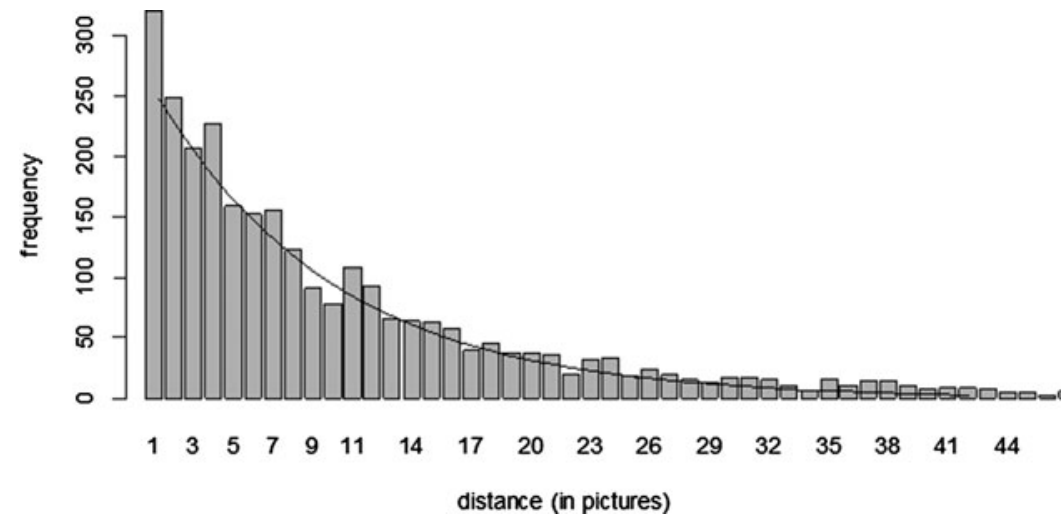

Fig. 3. Frequency distribution of pictures. The black line indicates the estimated random distribution.

geotagged in Berlin during 4 weeks in April 2015, some differences can be detected. First, the peak in the early mornings on weekdays is less pronounced in the Berlin data, as compared with the NSSI data. Furthermore, activity for NSSI pictures is much lower during the morning hours of weekdays, as Berlin data. While there is similar activity in the afternoon and early evening hours, the peak in the later evening hours is much more pronounced in NSSI than Berlin data.

\section{Characteristics of comments}

Of all 6651 comments posted on pictures of NSSI by other users than the person posting the picture, the most frequent type of comment were not directed at the user or picture directly, but were part of a general discussion $(n=3291,49.5 \%) .23 .5 \%(n=1562)$ of comments were empathic and $11.6 \% \quad(n=770)$ were warnings asking the user to stop the behavior. A few comments were offering help $(6.9 \%, n=462)$ or were abuse $(6.8 \%, n=450)$. Very few comments complimented the wound or picture $(0.5 \%, n=33) .1 .2 \%(n=83)$ of all comments did not have a meaningful content or were written in a language other than German or English.

\section{Association of comments with characteristics of pictures}

Across all 4 weeks, pictures directly depicting wounds generated around twice as many comments from other users than pictures not depicting wounds $(T=10.11, p$ $<0.001, \mathrm{M}_{\text {wound }}=4.1$, S.D. . $_{\text {wound }}=13.9 ; \mathrm{M}_{\text {No_wound }}=2.1$, S.D.No_wound $=9.3$ ).

There was also a significant association between wound grade and number of comments. Pictures showing very severe wounds generated more 
Table 1. Association of number of comments with characteristics of pictures

\begin{tabular}{lrllll}
\hline & & \multicolumn{2}{l}{$\mathrm{M}_{\text {Com }}$} \\
(s.D.Com $)$ & $F$ & $p$ \\
\hline Wound grade & & & 44.4 & $<0.001$ \\
$\quad$ Mild & 1118 & $2.6(7.2)$ & & \\
$\quad$ Moderate & 1351 & $3.7(10.9)$ & & \\
$\quad$ Severe & 357 & $10.3(29.5)$ & & \\
Wound type & & & 9.1 & $<0.001$ \\
Cutting & 2630 & $3.9(12.9)$ & & \\
Hitting & 35 & $1.6(2.0)$ & & \\
Others & 101 & $3.7(12.3)$ & & \\
Combination of & 52 & $13.8(41.7)$ & & \\
wounds & & & & \\
Body region & & & 0.50 & 0.68 \\
$\quad$ Extremities & 1988 & $4.74(16.2)$ & & \\
Torso & 47 & $2.7(3.7)$ & & \\
$\quad$ Head, neck & 13 & $1.6(3.2)$ & & \\
$\quad$ Mixed & 4 & $0.5(0.6)$ & & \\
Object & & & 0.24 & 0.79 \\
$\quad$ Razor blades & 114 & $3.3(6.9)$ & & \\
Other blades & 36 & $2.6(3.8)$ & & \\
Other object & 14 & $3.9(8.2)$ & & \\
& & & & \\
\hline
\end{tabular}

comments than moderate wounds and mild wounds (for details see Table 1).

There were no significant differences regarding number of comments between different body regions and different objects, respectively (for details see Table 1).

Wound grade was significantly associated with all types of comments. Generally, more severe wounds generated more comments, regardless of their content (see Table 2).

\section{Timely associations of NSSI pictures}

In order to detect possible effects of social contagion, different analyses were conducted. In a first step, the distribution of time differences between pictures was determined. For pictures showing wounds, the median was 363 s (25\%: 131 s, $75 \%$ : 806.5 s). The non-wound pictures had much lower time distances (median $=36$ s, $25 \%: 15 \mathrm{~s}, 75 \%$ : $79 \mathrm{~s}$ ), but, given that the percentage of wound pictures is roughly $10 \%$ of all pictures, the two distributions seem similar with roughly 10 times larger distances in wound pictures.

Secondly, the frequency distribution of the distances between wound pictures was calculated (i.e. how many pictures with NSSI hashtags, but not showing wounds were posted in-between pictures showing wounds). In case of social contagion, a higher number of smaller distances between pictures than a random distribution would be expected. However, as can be seen in
Fig. 3, the slope of the frequencies of distances between wound pictures followed approximately a random distribution. The higher number at distance 1 is explained by some users posting series of the same wound quickly in a row of 2 or more pictures $(N=55)$.

In a further analysis it was calculated whether the time difference to the next wound picture was correlated with the amount of comments a picture had, which was also controlled for number of followers a user had. There was a small positive significant correlation between amount of comments (Spearman- $\rho=$ $0.068, p<0.001$ ) as well as between comments by number of followers and time span between wound pictures (Spearman- $\rho=0.065, p=0.001$ ).

To account for possible effects of pictures being coincidentally posted at around the same time, these correlational analyses were repeated with pictures with at least $36 \mathrm{~s}$ (=Median) distance from each other, and also for an (arbitrarily chosen) interval of $60 \mathrm{~s}$, to allow for an appropriate time for users to react to pictures. These restrictions did only minimally change the size of correlation coefficients.

\section{Discussion}

This study is the first to systematically analyze a comprehensive sample of pictures showing NSSI wounds on Instagram. This study adds to the literature, as it investigated the second most popular social network in Germany after YouTube for NSSI content for the first time, evaluated a very large and comprehensive sample of all pictures being posted within a 4-weekperiod, was able to use timestamps of when pictures were posted, which is novel to research of NSSI online material, and was able to directly investigate the relationship of comments and pictures.

All pictures $(N=32182)$ associated with the most common German NSSI hashtags during a 4 week period in April 2016 were carefully rated and analyzed. In addition, corresponding comments and user profiles were investigated. Around $10 \%$ of all pictures associated with NSSI hashtags directly depicted wounds, which were most commonly caused by cutting on arms or legs. This is in line with a study by Seko \& Lewis (2016) who found that pictures with NSSI hashtags, which did not directly depict wounds were disseminated more widely than pictures directly depicting wounds.

Around $90 \%$ of NSSI pictures showed wounds that were rated as mild or moderate, while very severe wounds were rather rare. Furthermore, objects like razor blades were only depicted rarely.

Pictures with increasing wound grades and those showing different types of wounds generated significantly higher numbers of comments. Most comments 
Table 2. Association of type of comment with wound grade depicted

\begin{tabular}{|c|c|c|c|c|c|c|}
\hline Type of comment & Total $N$ & Mild wound $M$ (S.D.) & Medium wound $M$ (S.D.) & Severe wound $M$ (S.D.) & $F$ & $p$ \\
\hline Abusive & 450 & $0.07(0.6)$ & $0.17(1.2)$ & $0.40(1.9)$ & 11.6 & $<0.001$ \\
\hline Warning & 770 & $0.12(0.6)$ & $0.24(1.4)$ & $0.84(4.2)$ & 21.2 & $<0.001$ \\
\hline Discussion & 3291 & $0.59(2.8)$ & $1.11(6.4)$ & $3.11(11.98)$ & 21.2 & $<0.001$ \\
\hline Offering help & 462 & $0.07(0.33)$ & $0.17(0.69)$ & $0.42(1.58)$ & 28.1 & $<0.001$ \\
\hline Empathetic & 1562 & $0.40(2.03)$ & $0.52(2.48)$ & $1.15(4.55)$ & 10.71 & $<0.001$ \\
\hline Compliment & 33 & $0.00(0.1)$ & $0.01(0.1)$ & $0.03(0.2)$ & 3.98 & 0.019 \\
\hline
\end{tabular}

were general discussions of users or empathetic comments. Relatively few comments (compared with the overall amount of comments) were hostile or abusive, but also relatively few comments were directly offering help. However, comments with emotional content (like hostile ones or those empathetically offering help) might be the ones having most impact on users.

Characteristics of users were not analyzed further since around $80 \%$ were anonymous and the validity of indication of age or gender could not be verified. Interestingly, those participants who stated their age, were quite young (14.8 years, age range from 12 to 21 years). This is especially true when considering the minimum age required by Instagram being 13 years, and in theory participants could be of any age. This mean age corresponds well to the peak of prevalence rates in adolescence at around 15-16 years (Plener et al. 2015). However, it could be that younger Instagram users are more likely to state their age, as they might be less concerned about sharing information publicly. Furthermore, adolescents might be more concerned with their specific age (i.e. considering a large difference of being 14 or 16 years old due to legal restrictions), while older users might not be concerned with specifically mentioning their age. Another reason could be that most Instagram users are adolescents in general (Duggan \& Smith, 2013). Most users who stated their gender were female (around 90\%). This is also in line with studies showing more girls to be engaging in NSSI, and especially cutting (Bresin \& Schoenleber, 2015), and previous studies finding more online NSSI activity in females (i.e. Lewis et al. 2011).

Seko (2013) mostly identified superficial wounds in an analysis of pictures showing NSSI on Flickr, while these types of wounds were only depicted in around one third of pictures in the current study. This could point to an escalation in comparison with this earlier study, in a sense that users tend to post more severe injuries to be able to evoke reactions (as more severe wounds do result in more comments), or it could be explained by a tendency to share more severe material more openly.
Concerning timely trends of when pictures were posted, there was an insignificant peak of more pictures being posted on Sundays than on other days of the week. This peak could be explained by the lack of other activities available on Sundays (i.e. no school or work, and shops are closed on Sundays in Germany). This could also explain a large decline of pictures being posted between 8:00 and 1:00 hours on weekdays, since those are the most common school hours in Germany. One reason for differences of pictures being geotagged in Berlin and NSSI pictures during weekdays (with more steady activity in Berlin during the morning hours on weekdays) could be that the Berlin data might be influenced by tourists posting pictures. Another reason could be that pictures of NSSI might not be posted during the morning hours when users are usually at school or at work, while more neutral posts may well be posted during those hours. The largest peak of pictures being posted was in the evening between 20:00 and 22:00 hours on weekends and weekdays. This peak is much stronger for NSSI pictures than for general pictures being geotagged in Berlin. A reason for the pronounced peek for NSSI pictures could be that adolescents most often engage in NSSI in the evening and the urge for engaging in NSSI is highest in the evening. This is in line with recent findings that NSSI is associated with negative emotions during evening times (Turner et al. 2016). Also, posting NSSI pictures around the evening hours and early in the morning before going to school might be associated with rising levels of stress before having to go to school.

Regarding possible effects of social contagion, timerelated analyses did not point towards any effects of social contagion or reinforcement. First, no specific clustering of NSSI pictures being posted could be identified. Second, while a shorter time span in-between wound pictures with more comments would have pointed towards socially reinforcing mechanisms, no such effect was found. However, it has to be kept in mind that these are only first analyses with many un-known factors (e.g. someone might be triggered by a picture or might realize that NSSI pictures receive 
a large amount of attention, but a longer time might pass between this event, self-injuring, and posting the picture, which would then not be seen in our analyses).

One very important finding of this study is the association of increasing numbers of comments with increasing severity of wounds. This could - in contrast to the findings mentioned above - point towards a potentially socially reinforcing function of posting NSSI pictures online, as previously described by Lewis \& Seko (2016) and Dyson et al. (2016). As more severe wounds lead to significantly more comments, this might in turn lead to greater social reinforcement of individuals who post pictures of more severe wounds. This might also lead to individuals intentionally (or subconsciously) posting more severe pictures (and therefore performing more severe NSSI) as they experience a much greater response to severe pictures on other users' accounts or make the experience of gaining increasing interest by posting increasingly severe pictures. Furthermore, most comments (apart from neutral discussions and very few abusive comments) were empathic and supportive. This underlines the importance of social positive reinforcement as noted in Nock \& Prinstein's model (2004) and is in line with understanding NSSI as a way of seeking communication through behavior (Nock, 2008). However, it has to be kept in mind that posting NSSI pictures and actually engaging in NSSI are two different behaviors that might be influenced by different functions (i.e. NSSI might be serving mainly emotion regulation functions, while posting the picture might mainly be serving social functions).

\section{Limitations}

Although this study was performed with the utmost care, some limitations apply to the results. First of all, results cannot be generalized to international Instagram communities, as it was restricted to German language. However, results seem comparable with a recent study by Moreno et al. (2015), which focused on English hashtags and which also showed a large number of NSSI pictures to be present on Instagram. It has also been shown, that rates of NSSI in adolescents are comparable between German and the USA samples (Plener et al. 2009). Furthermore, this limitation can also be considered a strength, as it was therefore possible to analyze timely trends without confounds of different time zones, and differences in e.g. school- and shop-opening hours. Future studies would still be necessary to validate our results in different countries and cultures. Another limitation is the high rate of anonymous users, which did not make it possible to further evaluate their characteristics and cannot ensure whether pictures were actually taken by the users themselves. However, this is a limitation which applies to all studies performed online and cannot be solved in analyses focusing on data being made publicly available by users. This also reflects the real-world preference for NSSI to remain anonymous consistent with the secretive aspect of NSSI. Another aspect related to the secretive aspect of NSSI is that less obvious hashtags or pictures without hashtags may have been missed, as hashtags were identified if they were directly related to the hashtag 'cutting'. However, it is technically impossible to find all 'secret' hashtags related to NSSI, especially since those hashtags change rather quickly over time. Furthermore, inter-reliability was not calculated for the first initial identification of pictures. The interraterreliability for comments was rather low. This was due to the complicated nature of those comments, often containing different linguistic messages (i.e. a comment can be empathic and be offering help at the same time). Results on differences between types of comments should therefore be interpreted with care.

\section{Implications}

This study clearly shows that a large number of pictures of NSSI wounds are being posted daily on Instagram, even in a rather limited network of German hashtags. These figures occurred even though ten of the investigated 16 hashtags generated automatic warning messages. Some of these pictures showed very severe wounds, which can be disturbing to (especially younger) users of Instagram, and that those severe wounds generated more comments than pictures of mild wounds. Furthermore, as studies have shown that NSSI can be socially contagious (Jarvi et al. 2013), pictures on Instagram might put adolescents at risk to initiate NSSI or might be triggering for users already engaging in NSSI (Lewis \& Baker, 2011). Initiatives to remove those pictures off social media for those reasons have recently gone as far as users signing petitions to eliminate accounts with specific NSSI content (Ross, 2016). On the other hand, the reverse phenomena of inhibiting NSSI through engagement in a positive and supportive online community are also possible, as described in previous research (Lewis \& Seko, 2016).

Instagram reacted to pictures, comments or hashtags containing self-injurious or suicidal content by introducing suicide prevention tools. Users can report posts they find to be concerning and pop-up windows with support options appear when certain hashtags are searched for (e.g. links to helpful homepages or other resources). This is a first important step acknowledging responsibility for potentially harmful content. Given that NSSI wounds have been shown to be associated 
with current suicidal ideation (Burke et al. 2016), there is a potential for suicide prevention work in using pictures of wounds as identifier for high risk individuals potentially in need for further help. As we were able to show that especially those pictures showing more severe wounds generated more comments, preventative work might benefit from using machine learning to filter pictures showing more severe wounds, or to disable comment or like functions for those pictures. Furthermore, providing access to psychological (online) counseling through Instagram might be another step to use social media to prevent or diminish NSSI.

It is important for clinicians to be aware of online content of NSSI. Patients (and especially adolescent patients) should be asked about their online NSSI activity and how it influences their behavior. This can be important for therapy, when taking positively received comments on Instagram (i.e. being offered help) or negative comments (i.e. abusive comments) or triggering or socially reinforcing functions into account. These functions and also the effect of more severe pictures generating more comments could be reflected upon with the adolescent to make them more aware of the potential impact of their online NSSI activity on themselves and others. Two publications (Whitlock et al. 2007; Lewis et al. 2012) offer detailed insight on this topic for clinicians.

Overall, this study was able to show that NSSI pictures are prevalent on Instagram. Operators of social media platform need to be aware of this issue and respond appropriately.

\section{Acknowledgements}

This work was supported by the Volkswagen Foundation.

\section{Declaration of Interest}

All authors declare no conflict of interest with the potential to bias the work. TF is stakeholder of Reportage. FM; PLP has received research funding from the German federal ministry of research and education, the German federal agency for drugs and medical products, the German research foundation, the Baden-Wuerttemberg Foundation, Lundbeck pharmaceuticals and the Volkswagen Foundation.

\section{Ethical standards}

The authors assert that all procedures contributing to this work comply with the ethical standards of the relevant national and institutional committees on human experimentation and with the Helsinki Declaration of 1975, as revised in 2008.

\section{References}

Andalibi N, Ozturk P, Forte A (2017). Sensitive self-disclosures, responses, and social support on instagram: the case of \#depression. Paper presented at the ACM Conference on Computer Supported Cooperative Work and Social Computing, Portland, Oregon, USA.

Baker TG, Lewis SP (2013). Responses to online photographs of non-suicidal self-injury: a thematic analysis. Archives of Suicide Research 17, 223-235.

Bresin K, Schoenleber M (2015). Gender differences in the prevalence of nonsuicidal self-injury: a meta-analysis. Clinical Psychology Review 38, 55-64.

Burke TA, Hamilton JL, Cohen JN, Stange JP, Alloy LB (2016). Identifying a physical indicator of suicide risk: Non-suicidal self-injury scars predict suicidal ideation and suicide attempts. Comprehensive Psychiatry 65, 79-87.

Duggan M, Smith A (2013). Social media update 2013. Pew Research Center (http://pewinternet.org/Reports/2013/ Social-Media-Update.aspx).

Dyson MP, Hartling L, Shulhan J, Chisholm A, Milne A, Sundar P, Scott SD, Newton AS (2016). A systematic review of social media use to discuss and view deliberate self-harm acts. PLOS ONE 11, e0155813.

Feierabend S, Plankenhorn T, Rathgeb T (2015). Jim Studie (https://www.mpfs.de/fileadmin/files/Studien/JIM/2015/ JIM_Studie_2015.pdf).

Fischer T, Goldwich AD, Haentzschel O (2015). Instagram leaks. Neon 5, 16-21.

Guimaraes T (2014). Revealed: the demographic trends for every social network 2014 (http://www.businessinsider.de/ 2014-social-media-demographics-update-2014-9? $\mathrm{r}=\mathrm{US} \& \mathrm{IR}=\mathrm{T}$ ).

Jarvi S, Jackson B, Swenson L, Crawford H (2013). The impact of social contagion on non-suicidal self-injury: a review of the literature. Archives of Suicide Research 17, 1-19.

Landis JR, Koch GG (1977). The measurement of observer agreement for categorical data. Biometrics 33, 159-174.

Lewis SP, Baker TG (2011). The possible risks of self-injury web sites: a content analysis. Archives of Suicide Research 15, 390-396.

Lewis SP, Heath NL, Michal NJ, Duggan JM (2012). Non-suicidal self-injury, youth, and the Internet: what mental health professionals need to know. Child and Adolescent Psychiatry and Mental Health 6. doi:10.1186/1753-2000-6-13.

Lewis SP, Heath NL, St Denis JM, Noble R (2011). The scope of nonsuicidal self-injury on YouTube. Pediatrics 127, e552-e557.

Lewis SP, Seko Y (2016). A double-edged sword: a review of benefits and risks of online nonsuicidal self-injury activities. Journal of Clinical Psychology 72, 249-262.

Moreno MA, Ton A, Selkie E, Evans Y (2015). Secret society 123: understanding the language of self-harm on instagram. Journal of Adolescent Health 58, 78-84.

Nock MK (2008). Actions speak louder than words: An elaborated theoretical model of the social functions of 
self-injury and other harmful behaviors. Applied and Preventive Psychology 12, 159-168

Nock MK, Prinstein MJ (2004). A functional approach to the assessment of self-mutilative behavior. Journal of Consulting and Clinical Psychology 72, 885-890.

Plener P, Schumacher T, Munz L, Groschwitz R (2015). The longitudinal course of non-suicidal self-injury and deliberate self-harm: a systematic review of the literature. Borderline Personality Disorder and Emotion Dysregulation 2. doi:10.1186/s40479-014-0024-3.

Plener PL, Libal G, Keller F, Fegert JM, Muehlenkamp JJ (2009). An international comparison of adolescent non-suicidal self-injury (NSSI) and suicide attempts: Germany and the USA. Psychological Medicine, 39, 15491558.

R Development Core Team (2008). R: A Language and Environment For Statistical Computing. R Foundation for Statistical Computing: Vienna.
Ross RM (2016). Remove Emily LeRae Smith Off Of Facebook. London (https://www.change.org/p/markzuckerberg-remove-emily-lerae-smith-off-of-facebook). Accessed 15 December 2016.

Seko Y (2013). Picturesque wounds: a multimodal analysis of self-injury photographs on Flickr. Forum: Qualitative Social Research 14 (http://www.qualitative-research.net/index.php/ fqs/article/view/1935/3546).

Seko Y, Lewis SP (2016). The self-harmed, visualized, and reblogged: remaking of self-injury narratives on Tumblr. New Media \& Society. doi:1461444816660783.

Turner B, Cobb RJ, Gratz KL, Chapman AL (2016). The role of interpersonal conflict and perceived social support in nonsuicidal self-injury in daily life. Journal of Abnormal Psychology 125, 588-598.

Whitlock J, Lader W, Conterio K (2007). The internet and self-injury: what psychotherapists should know. Journal of Clinical Psychology 63, 1135-1143. 